\title{
The Utility of Liquid-Based Cytology in Salivary Gland Fine-Needle Aspirates: Experience of an Academic Institution
}

\author{
Jason M. Rarick Jay Wasman Claire W. Michael \\ Department of Pathology, University Hospitals Case Medical Center/Case Western Reserve University, \\ Cleveland, Ohio, USA
}

\section{Key Words \\ Salivary gland $\cdot$ Fine-needle aspiration $\cdot$ ThinPrep ${ }^{\circledR}$. \\ Conventional smear $\cdot$ Liquid-based cytology}

\begin{abstract}
Objective: To review the morphological features of salivary gland fine-needle aspirates (FNA) on ThinPrep ${ }^{\circledR}$ (TP) preparations. Emphasis is placed on the commonalities and specific differences between TP and conventional smear (CS) preparations. Study Design: The cytology and surgical pathology archives were searched for 'salivary' or 'parotid' at our institution from 2003 to 2013 for cases processed by TP and with a range of specified diagnoses and surgical followup. These cases were reviewed by the authors. A review of the known literature was also performed. Results: Morphological features and artifacts were noted for the most common salivary gland lesions. General features noted in the literature were identified, along with novel features identified on our review of cases. Emphasis was placed on cellular alterations, extracellular differences and architectural changes. Conclusions: The multitude of both benign and malignant lesions coupled with the unique artifacts encountered on TP makes salivary gland FNAs difficult for most practicing pathologists and cytopathologists. For this reason, many
\end{abstract}

laboratories have been reluctant to implement TP preparations for these lesions. With knowledge of the specific artifacts and differences on TP compared to CS, TP can be used with confidence without a compromise in diagnostic accuracy for adequately cellular samples.

c) 2014 S. Karger AG, Basel

\section{Introduction}

Liquid-based cytology (LBC) was originally developed for Pap tests and has subsequently been increasingly popular in many laboratories as a diagnostic modality for nongynecological specimens in the USA and many other countries. This methodology has several advantages over conventional smears (CS), including the following: (1) more standardized processing, especially with samples received from outside clinics or shipped from a distance to centralized laboratories; (2) decreased screening area; (3) decreased obscuring material such as blood, inflammation and mucus; (4) lack of air-drying artifacts; (5) a more monolayer cellular surface that is easier to screen; (6) consistently well-preserved cells, and (7) the potential for automated screening in LBC specimens [1-4]. Because of the many advantages of $\mathrm{LBC}$, the technique has

\section{KARGER}

E-Mail karger@karger.com www.karger.com/acy
(C) 2014 S. Karger AG, Basel

0001-5547/14/0586-0552\$39.50/0 
Table 1. General artifacts related to TP methodology

\begin{tabular}{|c|c|}
\hline Technique & Resulting artifacts \\
\hline $\begin{array}{l}\text { CytoLyt and } \\
\text { PreservCyt }{ }^{\circledR} \\
\text { fixation } \\
\text { (methanol- } \\
\text { based with } \\
\text { mucolytic } \\
\text { and hemolytic } \\
\text { agents) }\end{array}$ & $\begin{array}{l}\text { - Only fixed preparations with Pap stain } \\
\text { - No air-dried preparations available } \\
\text { - Nucleoli are more conspicuous } \\
\text { - Cells appear smaller } \\
\text { - Cytoplasm has denser quality } \\
\text { - Less bare nuclei } \\
\text { - Hemolyzed blood leaving behind few mixed } \\
\quad \text { inflammatory cells } \\
\text { - } \\
\text { - } \text { Alter mucus and other extracellular matrix } \\
\text { Clear background }\end{array}$ \\
\hline $\begin{array}{l}\text { Cell dispersion } \\
\text { (gentle } \\
\text { agitation) }\end{array}$ & $\begin{array}{l}\text { - Breakdown of large fragments into smaller } \\
\text { and less complex groups } \\
\text { - Increase number of single cells in } \\
\text { background (pseudo-dyscohesion) }\end{array}$ \\
\hline $\begin{array}{l}\text { Cell collection } \\
\text { (filtration) }\end{array}$ & $\begin{array}{l}\text { - Loss of small cells such as lymphocytes and } \\
\text { myoepithelial cells } \\
\text { - } \text { Loss of tumor diathesis } \\
\text { - Large fragments may not get through the } \\
\text { filter } \\
\text { - } \text { Filter may be blocked by excessive blood or } \\
\text { inflammation, resulting in ring effect } \\
\text { - Controlled cell density with minimal over } \\
\text { lapping }\end{array}$ \\
\hline $\begin{array}{l}\text { Cell transfer } \\
\text { (positive air } \\
\text { pressure) }\end{array}$ & $\begin{array}{l}\text { - Cells and clusters are artificially flattened } \\
\text { - More flattening at the periphery (ring effect) } \\
\text { - Cells are denser at the periphery than at the } \\
\text { center of the preparation } \\
\text { - Small cells are identified mostly at the } \\
\text { periphery of the preparation }\end{array}$ \\
\hline
\end{tabular}

been increasingly adopted by many laboratories for nongynecological specimens, particularly exfoliative samples. However, the utilization of LBC for fine-needle aspiration (FNA) has been controversial, particularly in sites such as the salivary gland where the architecture, extracellular matrix material and inflammatory component play a major role in the diagnosis.

Despite the advantages of LBC, several morphological alterations are notable. Most of the published literature on the application of LBC for nongynecological samples is focused on ThinPrep ${ }^{\circledR}$ (TP) and very little has been published on SurePath. While there is a large body of literature discussing findings in nongynecological specimens by TP, the literature on salivary gland FNA biopsies using this technique is extremely limited. As noted by previous reports [1-3], LBC introduces new artifacts that alter the cellular, architectural and extracellular matrix appearance (table 1). The authors emphasized the need for becoming familiar with those artifacts prior to full implementation of this new technology for patient care. At the University Hospitals Case Medical Center we implemented the use of TP for gynecological and nongynecological specimens in 1998. In this review we discuss our experience of the use of LBC in FNA of the salivary glands.

\section{Nonneoplastic Aspirates}

\section{Normal Acinar Structures}

Few groups of acinar cells, either serous or mucinous, with small ductal groups or stromal fragments may be encountered due to sampling error or coexistence with other lesional findings. They are usually seen as ball-like single clusters and rarely in groups. Their presence and identity are easy to recognize on TP. The cytoplasm appears denser with less conspicuous vacuoles and the clusters tend to be compressed or flattened. When seen in sialadenosis, the preparations are highly cellular and comprised of numerous grouped clusters with a pattern resembling a bunch of grapes, similar to those on CS but with less complexity (fig. 1). The cells are usually slightly larger and with more vacuolated cytoplasm than the resting acinar cells. Because of the absence of manual spreading and wet fixation, the cytoplasm is not fragile as in CS and consequently bare nuclei are markedly reduced.

TP features:

(1) Variable cellularity (low in normal salivary gland, high in sialadenosis)

(2) Predominance of acinar groups with rare adipose and ductal epithelial fragments

(3) Acinar groups with intact cytoplasm and no bare nuclei

(4) Reduced complexity of cellular groups

\section{Benign Cystic Lesions}

Most benign cysts are either retention cysts or mucoceles. The TP slide is usually of low cellularity and consists mainly of macrophages in a clear background even in the case of a mucocele since the mucoid material will be altered/reduced. Epithelial cells, when present, are easily identified.

TP features:

(1) Predominance of macrophages and possible cyst-lining cells

(2) Cleaner background with minimal-to-no cellular debris 


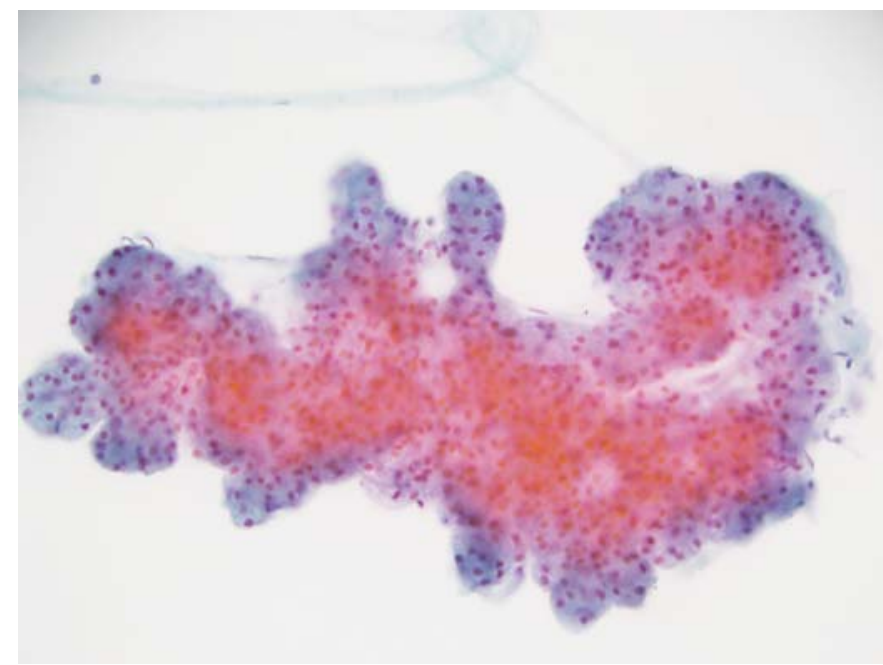

Fig. 1. Sialadenosis. This sheet of salivary gland acinar cells appears as bunches of grapes. The acinar cells contain abundant, granular to finely vacuolated cytoplasm and small, uniform eccentrically placed nuclei (Papanicolaou, TP, $\times 400$ ).

\section{Lymphocyte-Rich Lesions}

In chronic sialadenitis and Sjögren's syndrome, the TP slide is of low cellularity and contains few ductal groups in a clear background, with rare lymphocytes either infiltrating the cellular groups or noted mainly at the periphery of the preparation. Additionally, the lymphocytes tend to aggregate together associated with fibrin strands.

In aspirates of HIV and non-HIV-associated benign lymphoepithelial cysts, the TP is more cellular and contains multinucleated cells and/or epithelial lining cells in a background of lymphocytes. In aspirates of Warthin tumor, the TP typically contains a random representation of small oncocytic groups and scattered lymphocytes, sometimes in aggregates, that are frequently away from the clusters or populating the periphery of the preparation (fig. 2a, b). Those lymphocytes are best identified by examination at low magnification. The background is clear and contains few histiocytes but no mucoid material or acellular debris. On occasional cases we identified that the cytoplasm of the oncocytic cells may be deceptively vacuolated even in the correlating cell block. As in CS, some Warthin tumor cases may present predominantly with an oncocytic component where the differential with an oncocytic neoplasm is considered or exclusively with lymphocytes where a lymph node or lymphoma are considered.

Aspirates of lymphoproliferative lesions arising in myoepithelial sialadenitis or an intraparotid lymph node
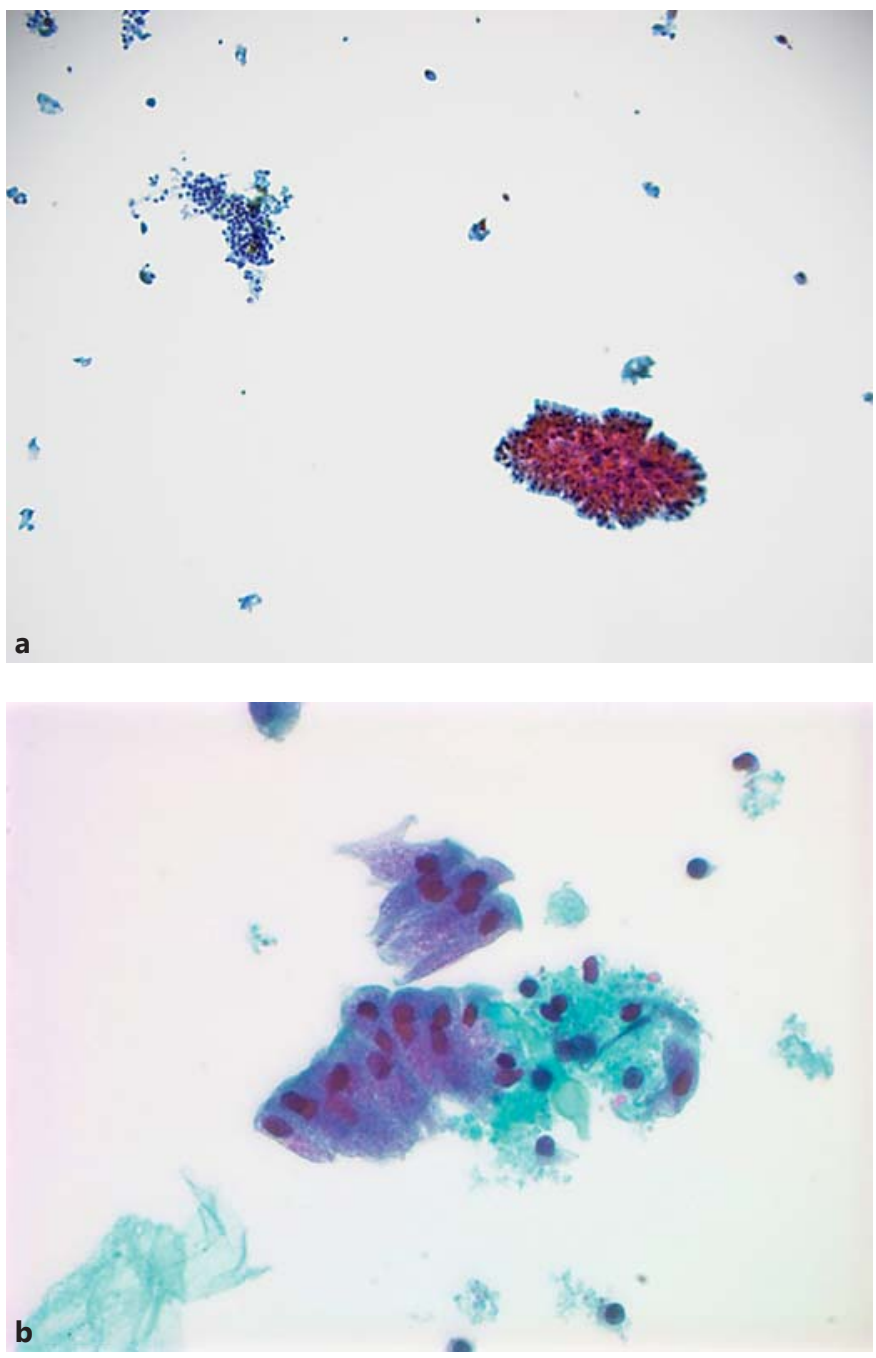

Fig. 2. Warthin's tumor. a A cohesive group of oncocytic epithelial cells (lower right) in a clean background and scattered lymphocytes in smaller clusters and individually are present (Papanicolaou, TP, $\times 200)$. b On higher power, the abundant granular cytoplasm present in the oncocytes is evident. The nuclei of the oncocytic cells are round, uniform and crowded within this cohesive group. A few scattered lymphocytes are present at the periphery of the group associated with a small amount of fibrin (Papanicolaou, $\mathrm{TP}, \times 1,000)$

are highly cellular and contain numerous lymphocytes and a scant or absent epithelial component. Also, tingible body macrophages are very easy to identify. However, because the cells are smaller in size, it is difficult to evaluate the nuclear detail of the different lymphocyte types, making a cell block preparation and flow cytometry even more necessary to evaluate for lymphoma. 

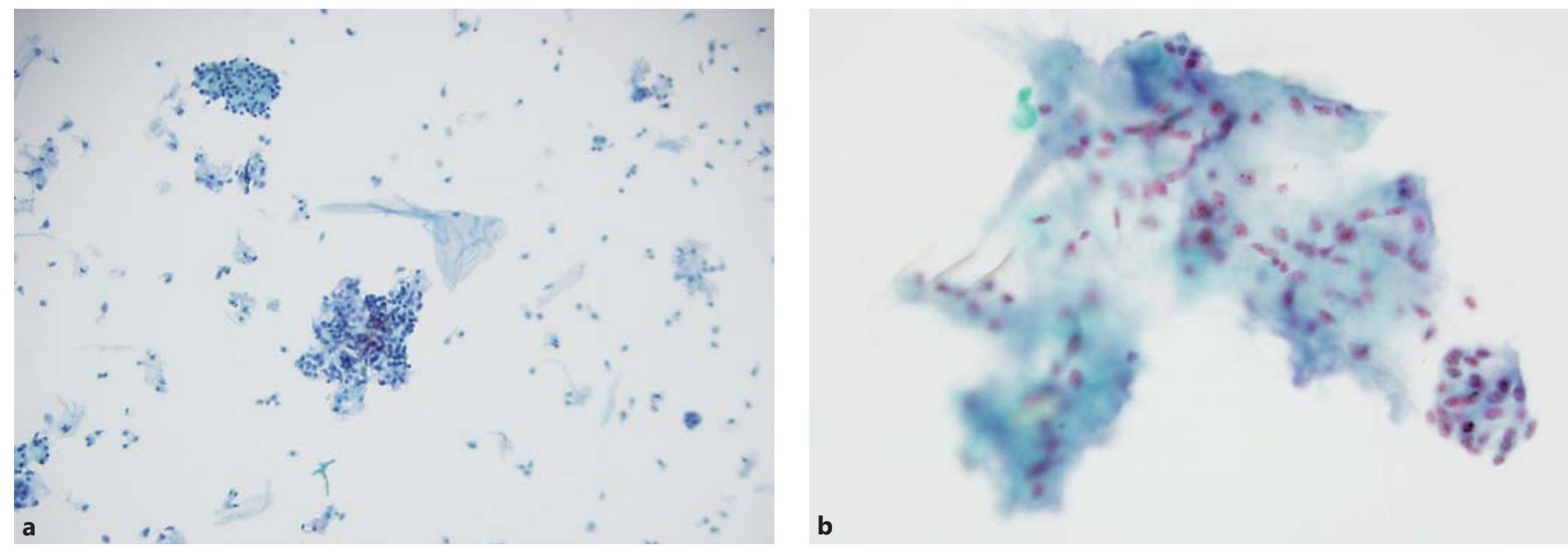

Fig. 3. Pleomorphic adenoma. a This moderately cellular specimen contains epithelial-myopeithelial cells in sheets with scattered isolated epithelial and myoepithelial cells present in the background. Light blue-gray feathery matrix material is also seen in the background (Papanicolaou, TP, $\times 00$ ). b On higher power, myoepithelial cells with bland, uniform, oval nuclei are dispersed within the stromal matrix material (Papanicolaou, TP, $\times 1,000)$.

TP features:

(1) Smaller groups of representative epithelial cells

(2) Reduced lymphocytes, acellular debris and mucoid material

(3) Lymphocytes more obvious at the periphery

(4) Lymphocytes often entrapped within fibrin, making small lymphocytic aggregates

(5) Highly cellular specimens containing mostly lymphocytes in lymphoproliferative lesions and intraparotid lymph nodes

(6) Lymphocyte cellular detail often lost in TP specimens, necessitating ancillary studies

\section{Neoplasms with Extracellular Matrix}

This category includes a wide range of neoplasms with mucinous, myxoid, hyaline or collagenous matrix. While the TP process alters both the quality and quantity of such a matrix, we noticed that mucin and watery myxoid material are affected the most. As the matrix gets denser in quality, e.g. dense myxoid or chondroid, it tends to withstand the TP process better.

\section{Pleomorphic Adenoma and Carcinoma ex \\ Pleomorphic}

The cellularity of these aspirates is widely variable. Low cellular aspirates are more problematic to recognize as the TP slide contains few small epithelial sheets frequently detached from the stroma that are less complex than those typically seen in CS. Scattered individual epi- thelial and myoepithelial cells can be identified in the background. The more cellular the specimen or dense the stroma, the easier it is to recognize. Loose stroma is severely compromised and appears as rare fragments of porous material resembling moth-eaten leaves. In more cellular aspirates, the stroma is easily recognized as it associates with the epithelial/myoepithelial component as traditionally seen on CS (fig. 3a, b). In the few examples of carcinoma ex pleomorphic that we encountered, the TP was very similar in appearance to that of the pleomorphic adenoma except for the presence of few cells that exhibited a high degree of atypia and triggered a suspicious diagnosis.

TP features:

(1) Smaller, less complex two-dimensional clusters of epithelial cells

(2) Increased numbers of background individual cells, derived from both epithelial and myoepithelial cells

(3) Myxoid matrix altered in both quality and quantity

(4) Cells with high degree of atypia may be encountered in carcinoma ex pleomorphic

\section{Basaloid Neoplasms}

Basaloid neoplasms comprise a broad category that includes cellular pleomorphic adenoma, basal cell adenoma, basal cell adenocarcinoma, and adenoid cystic carcinoma. Adenoid cystic carcinoma will be discussed in more detail separately. Further classification can be difficult-to-impossible on cytology alone. 


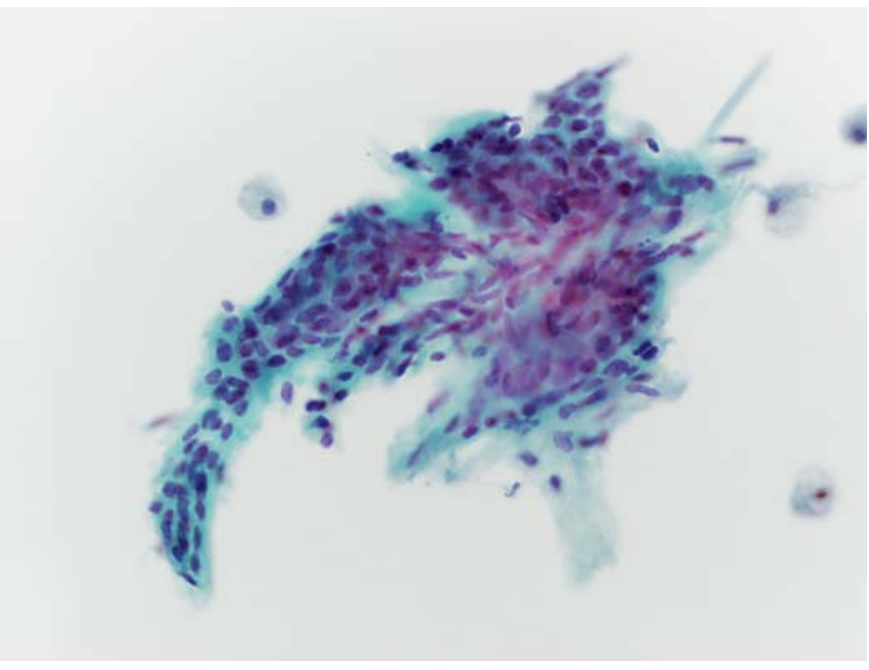

Fig. 4. Basal cell adenoma, membranous type. An irregularly shaped, crowded, cohesive cluster of small, basaloid cells with uniform, oval, hyperchromatic nuclei is seen with a few scattered macrophages in the background. A layer of dense, hyaline material surrounds the basaloid cells (Papanicolaou, TP, ×600).

In basaloid neoplasms, the TP slide is usually cellular and contains variably sized groups of basaloid cells. Because of the fragmentation of these clusters, no specific pattern, e.g. trabecular or tubular, can be easily identified. The clusters are generally irregular and may have sharp edges. Representation of stroma depends on its nature in the lesion itself. Frequently, the basaloid cells are embedded and surrounded by the dense matrix. The features overlap greatly among these entities and it may be impossible to further classify them. We encountered a case of basal cell adenoma - membranous type - which presented as clusters of basaloid cells either with rounded and smooth or sharp and irregular borders, surrounded by a thin layer of dense hyaline material (fig. 4). In a case of canalicular adenoma, the TP slide contained several clusters with basaloid epithelial cells forming Chinese-like characters embedded in the stroma. While the difference was subtle, we could appreciate the fine reticular quality of the cords within the stroma. Those cellular clusters and cords were distinctly outlined by crowded palisade columnar cells (fig. 5). Other examples of basal cell adenoma and carcinoma presented with cellular preparations containing groups of basaloid cells without any other characteristic features. Background histiocytes are seen in lesions with a cystic component. A solid variant of adenoid cystic carcinoma presents similarly with small fragments of basaloid cells almost indistinguishable from the
Fig. 5. Basal cell adenoma, canalicular type. Cohesive clusters of small, basaloid cells show uniform, oval, hyperchromatic nuclei. Peripherally palisading nuclei are prominent in some areas. Only a small amount of feathery stromal matrix material is present in this example (Papanicolaou, TP, $\times 600)$.

above neoplasms. However, we found that the cells tend to be relatively more crowded and irregular with few discernible angulated nuclei (fig. 6).

TP features:

(1) Small ill-defined fragments of basaloid cells

(2) The basaloid cells are usually embedded in the hyaline matrix

(3) Palisaded columnar cells outlining the clusters may be seen in certain types

(4) Diagnosis of specific entities often not possible on TP slide alone

\section{Adenoid Cystic Carcinoma}

Cellular aspirates of adenoid cystic carcinoma are not difficult to diagnose on TP, although one must be aware of differences compared to CS. The traditional complex fragments seen in CS are fragmented in TP and appear as small clusters with simple architecture. The hyaline cylindrical matrix can be seen surrounded by the small cells or as completely detached balls in the background. The matrix tends to have a more delicate quality than that seen on CS. Rarely, branching groups with central cylindrical matrix are seen (fig. 7). More individual cells with a high nuclear-to-cytoplasmic ratio are noted in the background of TP slides. 


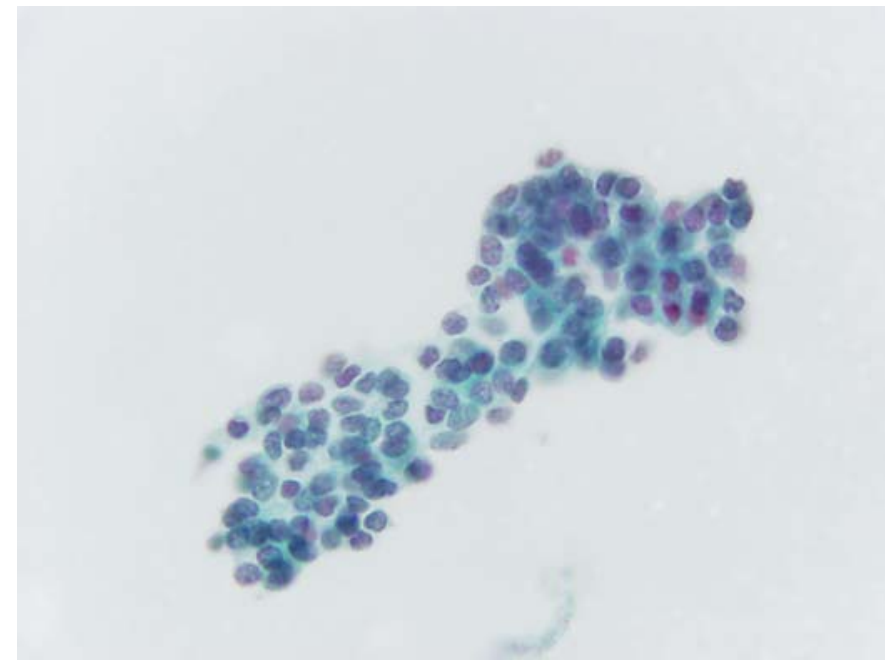

Fig. 6. Adenoid cystic carcinoma, solid variant. Similar to basal cell adenoma, this solid variant of adenoid cystic carcinoma shows a cohesive cluster of basaloid cells. There is nuclear crowding and occasional angulated nuclei; extracellular matrix material is not identifiable (Papanicolaou, TP, $\times 1,000$ ).

TP features:

(1) Smaller, less complex basaloid epithelial fragments present with less developed epithelial latticework

(2) More individual cells in background

(3) Acellular cylindrical stroma present, though less developed

\section{Epithelial Myoepithelial Carcinoma}

The TP slide is cellular and contains numerous variable-sized fragmented sheets of cells frequently associated with extracellular matrix. The characteristic twocell populations (epithelial inner lining cells and myoepithelial outer cells) can be recognized; they are not organized within the sheets but rather admixed. However, a population of small cuboidal-to-columnar cells admixed with larger cells with more visible cytoplasm can be easily recognized. Performing immunostains can clearly highlight both the epithelial and myoepithelial components and help establish a definitive diagnosis. Extracellular matrix, including hyaline basement membrane material, is present variably. It can be seen as globular structures mimicking those of adenoid cystic carcinoma or fragments of amorphous material mimicking the myxoid material seen in pleomorphic adenoma. Generally, the matrix is seen within the clusters or loosely associated with it. However, it is not distinctly surrounded by the cells as in adenoid cystic carcinoma or

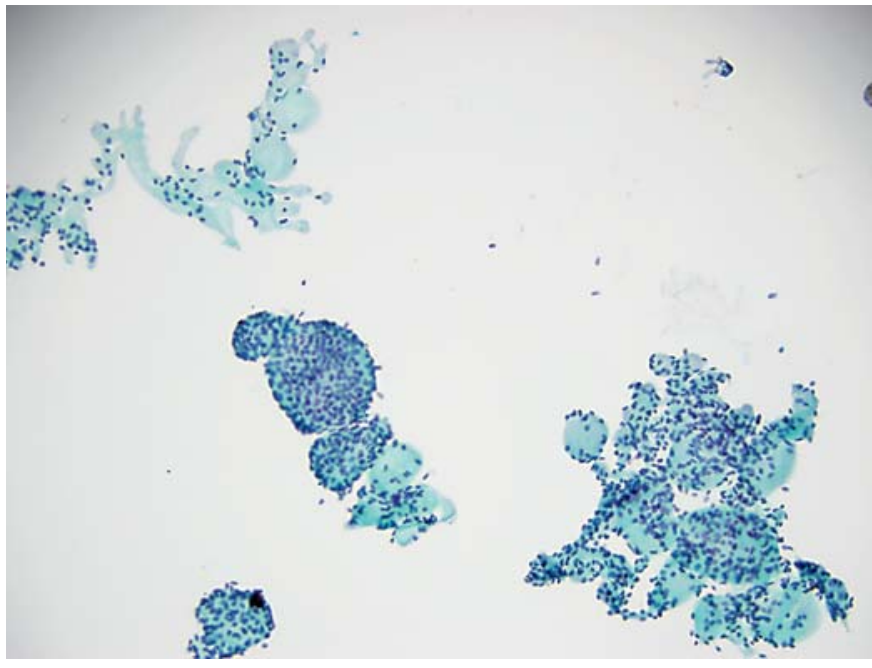

Fig. 7. Adenoid cystic carcinoma, cribriform pattern: branching sheets and clusters of small, basaloid cells contain cylindrical hyaline matrix material. Note how the basaloid cells surround the round globules of hyaline material, typical of adenoid cystic carcinoma (Papanicolaou, TP, ×200).

intermixed with the cells as in pleomorphic adenoma. Occasional sheets with distinctly more atypical cells can also be seen (fig. 8).

TP features:

(1) Variable-sized fragmented sheets of cells

(2) Two-cell populations (epithelial and myoepithelial cells)

(3) Basement membrane-like hyalinized material in globular structures, with thin amorphous fragments within the clusters or loosely associated with them

\section{Mucoepidermoid Carcinoma}

Because mucin tends to be significantly altered by TP, mucoepidermoid carcinoma, particularly low-grade lesions, can be difficult to recognize. A TP slide of a lowgrade mucoepidermoid carcinoma may contain either few glandular cells with very subtle atypia in a background of histiocytes or a mixture of glandular cells merging with intermediate cells with squamoid appearance. Glandular cells with a slightly high nuclear-to-cytoplasmic ratio and small prominent nucleoli may be seen occasionally. The glandular cells have somewhat denser cytoplasm than seen in CS and the mucin may completely disappear or be difficult to identify with certainty. A helpful clue is to look for the mucin trapped within the cellular groups or as intracytoplasmic vacuoles (fig. 9). 


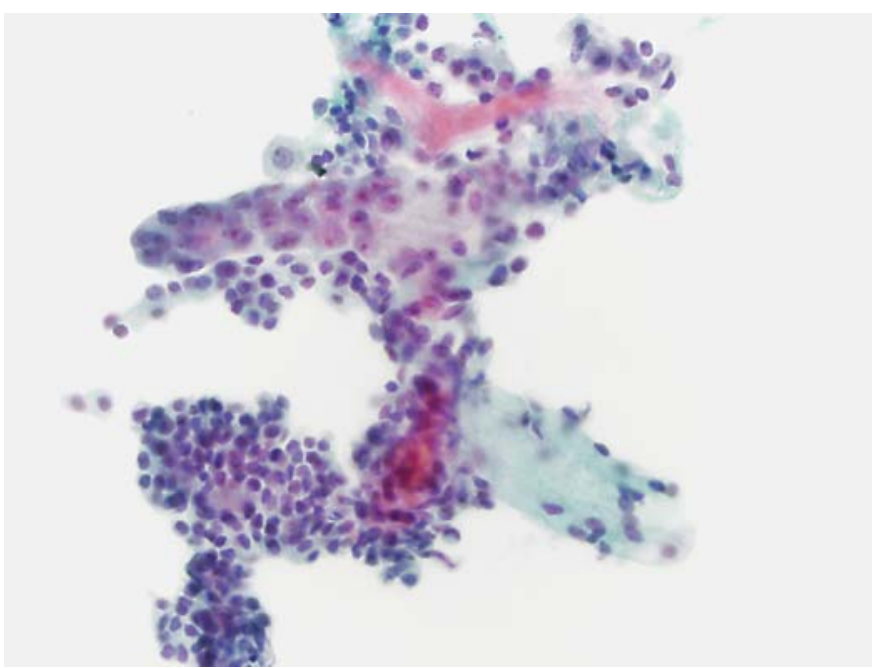

Fig. 8. Epithelial myoepithelial carcinoma. The dual cell population is easily identified; the inner layer of larger epithelial cells shows enlarged nuclei with open chromatin and small prominent nuclei. The surrounding myoepithelial component is comprised of small basaloid cells with smaller, uniform, oval, hyperchromatic nuclei (Papanicolaou, TP, $\times 600$ ).

High-grade cases show features of high-grade carcinoma and tend to present with numerous intermediatetype cells and malignant squamous cells. These two cell types are easily identified, especially the keratinizing squamous cells when present.

TP features:

(1) Mucinous cells with minimal atypia and more individual cells

(2) Extracellular mucin extremely rare and difficult to identify

(3) Intracellular mucin droplets or droplets entrapped within clusters may be identified

(4) Mixture of glandular, intermediate and squamous cells

(5) High-grade lesions show significant cytological atypia

\section{Tumors with Predominantly Epithelial Component}

\section{Oncocytoma}

The TP slide is cellular and contains sheets of oncocytic cells in a background of numerous single oncocytes. The cells are relatively larger than those encountered in Warthin tumor or oncocytic hyperplasia and possess abundant intensely granular cytoplasm (mitochondriarich) and vesicular nuclei, frequently with prominent nu-

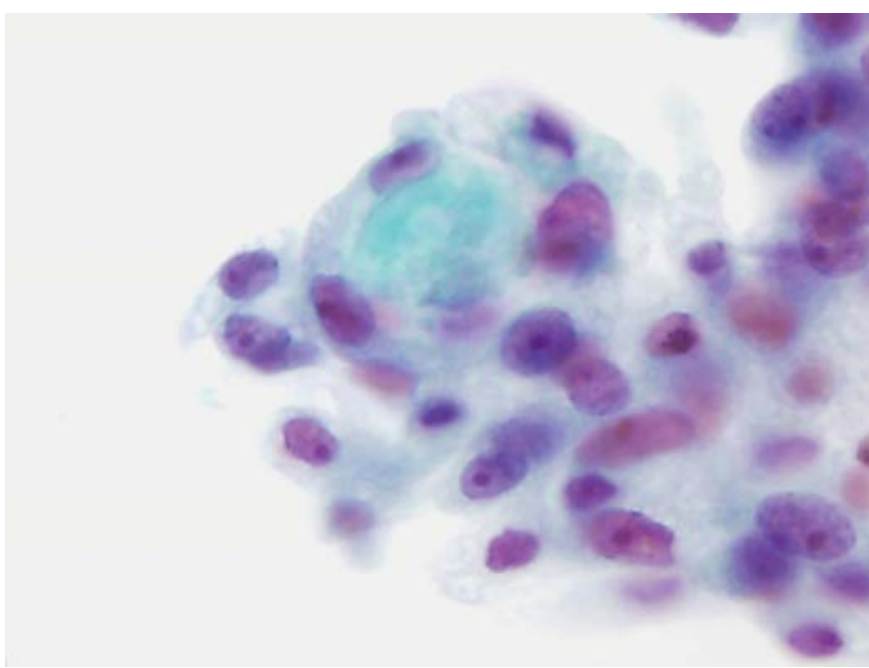

Fig. 9. Mucoepidermoid carcinoma, low grade. This cluster of epithelial cells is mildly atypical with finely vacuolated cytoplasm, mildly enlarged nuclei, and prominent nucleoli. Entrapped mucinous material is present within this cell cluster (Papanicolaou, TP, $\times 1,000)$.

cleoli. The background is clean with no histiocytes or lymphocytes. The oncocytes on TP may appear markedly vacuolated as a result of fixation artifact.

Depending on the architectural pattern of the oncocytoma, vague papillae may be noted. In some cases the clusters may have scalloped borders and mimic benign acinar groups. Indeed, we encountered a case that was originally misinterpreted as benign acini (fig. 10).

TP features:

(1) Cellular preparations containing fragmented sheets of oncocytic and single cells

(2) Occasional cytoplasmic vacuoles (fixation artifact)

(3) May form vague papillae or clusters with scalloped borders

\section{Acinic Carcinoma}

The TP slide is usually cellular and contains a mixture of small sheets and single acinar cells with rich cytoplasmic granules and no associated ductal groups. Numerous histiocytes may also be noted in tumors with a cystic component. The cells tend to be larger and relatively more crowded and overlapping (less organized) compared to benign acini (fig. 11). Contrary to CS, bare nuclei are markedly reduced. Low cellular aspirates can be easily mistaken for benign acinar groups. 


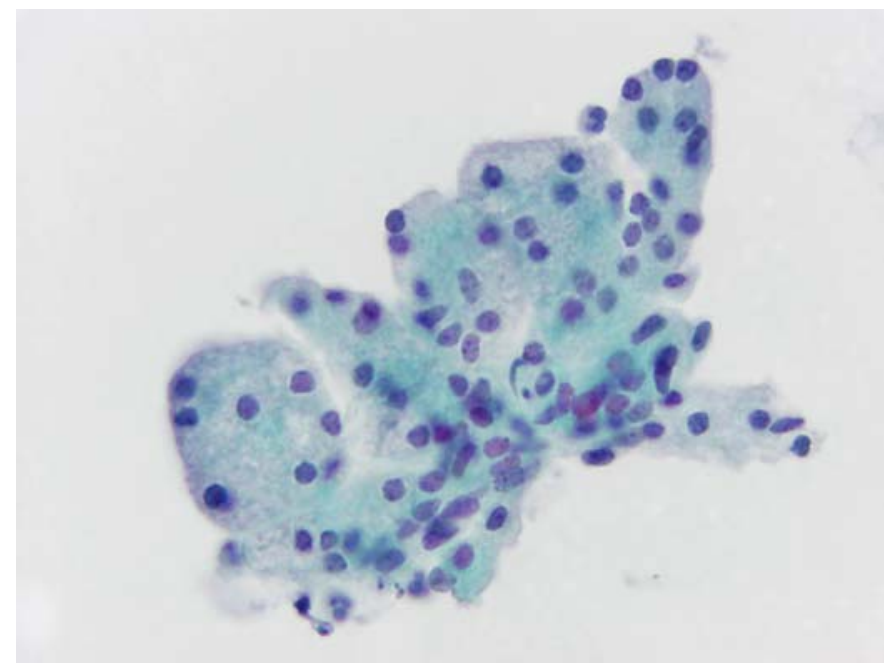

Fig. 10. Oncocytoma. Cohesive clusters of cells with a low nuclearto-cytoplasmic ratio are present. The cells contain abundant cytoplasm that is granular to finely vacuolated. The nuclei are small, round to oval, and some contain small nucleoli. These cells may be difficult to distinguish from acinar cells on TP (Papanicolaou, $\mathrm{TP}, \times 600)$.

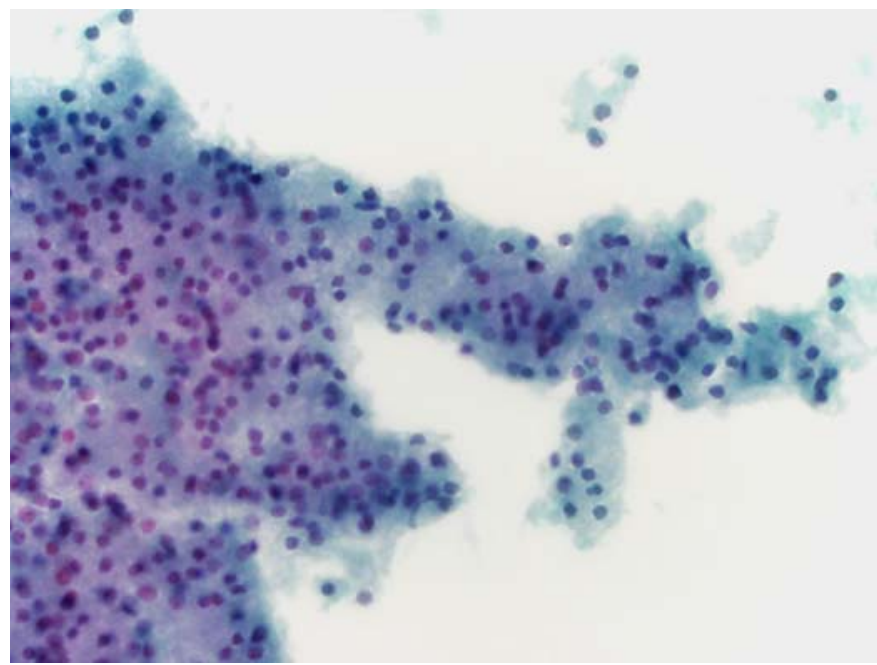

Fig. 11. Acinic cell carcinoma. A large sheet and scattered small clusters of cells with low nuclear to cytoplasmic ratios and abundant granular cytoplasms are present. The nuclei are round to oval and uniform. Note the resemblance to normal acinar cells and the absence of ductal type epithelium (Papanicolaou, TP, $\times 600$ )
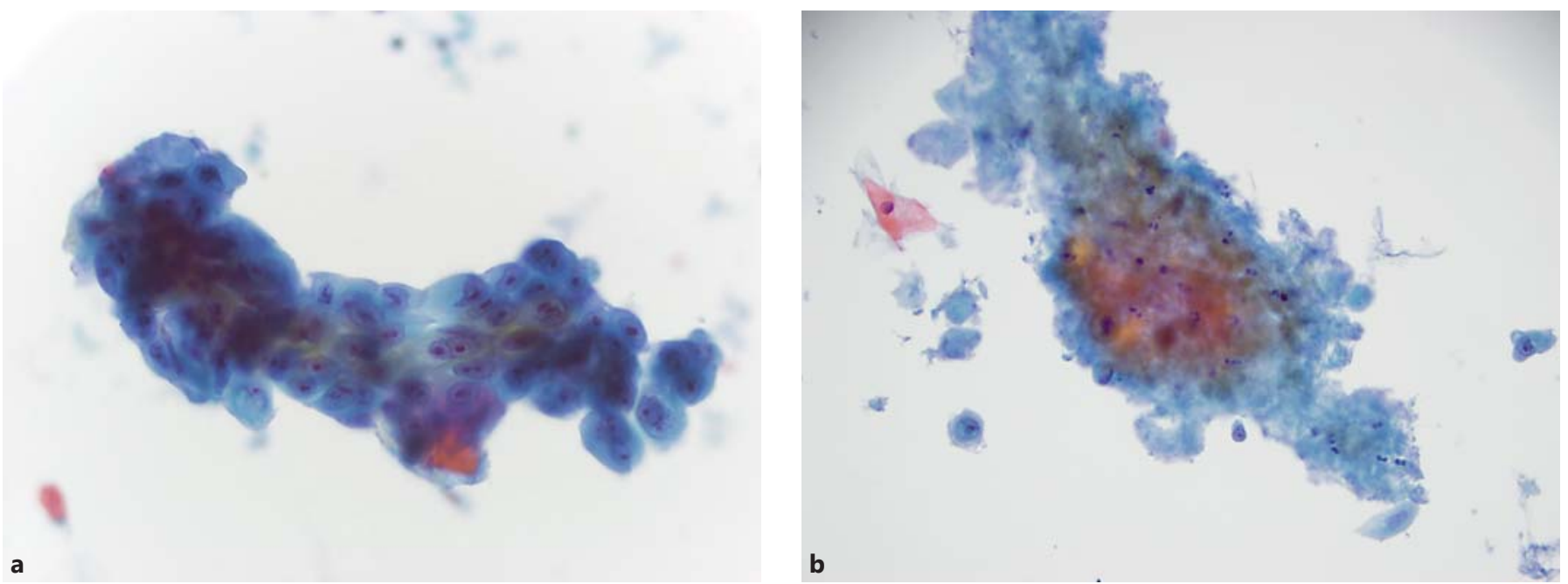

Fig. 12. Squamous cell carcinoma. a This group of epithelial cells contains dense, waxy cytoplasm and high nuclear to cytoplasmic ratios. The nuclei are large with irregular nuclear contours and large, prominent nucleoli. While not entirely specific, the features are characteristic of squamous cell carcinoma (Papanicolaou, TP, $\times 600$ ). b Cellular and nuclear debris trapped within fibrin is seen in the center, while rare, isolated malignant squamous cells are adjacent (Papanicolaou, $\mathrm{TP}, \times 400)$.

\section{TP features:}

(1) Moderate-to-cellular TP slide

(2) Cohesive and dyscohesive acinar cells with disordered arrangement

(3) No ductal cell groups

(4) Inconspicuous bare nuclei

\section{Salivary Duct Carcinoma}

The TP is cellular and contains numerous small- to medium-sized groups of monotonously appearing polygonal cells with abundant cytoplasm and central nuclei with prominent nucleoli. The cells are significantly pleomorphic. As is the case with TP, tumor diathesis is mark- 
Table 2. Tips for circumventing artifacts in TP-processed aspirates

\begin{tabular}{|c|c|}
\hline Problem & Tip \\
\hline $\begin{array}{l}\text { Distinguishing true } \\
\text { inflammation from } \\
\text { peripheral blood } \\
\text { elements }\end{array}$ & $\begin{array}{l}\text { - Peripheral blood elements are of mixed } \\
\text { cell type containing both neutrophils } \\
\text { and lymphocytes }\end{array}$ \\
\hline $\begin{array}{l}\text { Reduced } \\
\text { lymphocytes }\end{array}$ & $\begin{array}{l}\text { - Look for lymphocytes entrapped within } \\
\text { the clusters or the fibrin clots } \\
\text { - Look at the periphery of the preparation } \\
\text { where they tend to concentrate }\end{array}$ \\
\hline $\begin{array}{l}\text { Reduced tumor } \\
\text { diathesis }\end{array}$ & $\begin{array}{l}\text { - Look for nuclear debris and old blood } \\
\text { entrapped within fibrin clots }\end{array}$ \\
\hline $\begin{array}{l}\text { Markedly decreased } \\
\text { mucin }\end{array}$ & $\begin{array}{l}\text { - Look for intracellular mucin } \\
\text { - } \text { Look for mucin entrapped within cell } \\
\text { clusters or fibrin clots }\end{array}$ \\
\hline Altered matrix & $\begin{array}{l}\text { - Better identified within larger groups } \\
\text { - Frequently loosely detached in the } \\
\text { background }\end{array}$ \\
\hline $\begin{array}{l}\text { Variable cellularity } \\
\text { across the } \\
\text { preparation }\end{array}$ & $\begin{array}{l}\text { - Scan the slide at low magnification } \\
\text { since the center tends to have less } \\
\text { clusters and cells than the periphery }\end{array}$ \\
\hline Ring effect & $\begin{array}{l}\text { - This results from blocked filter holes } \\
\text { - Specimen may need reprocessing to } \\
\text { eliminate obscuring blood and repeat } \\
\text { when relevant }\end{array}$ \\
\hline $\begin{array}{l}\text { Cells appear flat or } \\
\text { not well preserved }\end{array}$ & $\begin{array}{l}\text { - Avoid evaluating cells that are at the } \\
\text { periphery, which are usually subjected to } \\
\text { pressure by the ring }\end{array}$ \\
\hline
\end{tabular}

edly reduced and mainly seen as debris entrapped within fibrin clots.

TP features:

(1) Cellular TP slide containing groups of atypical cells

(2) Reduced diathesis compared to CS

\section{Squamous Cell Carcinoma}

A squamous cell carcinoma primary tumor of the salivary gland is uncommon; it usually represents a metastasis or is the result of direct invasion into the salivary gland from a head or neck primary tumor. A well-differentiated squamous cell carcinoma is highlighted by dyscohesive dyskeratotic cells with irregular hyperchromatic nuclei and dense orangeophilic cytoplasm which are nicely highlighted by the TP Pap stain. A poorly differentiated squamous cell carcinoma often shows clusters of cohesive atypical cells with a high nuclear-to-cytoplasmic ratio and large nuclei with prominent nucleoli. Contrary to CS, the characteristic tumor diathesis of squamous cell carcinoma is markedly reduced to nonexistent on TP. The best clue for this diathesis is recognizing the cellular and nuclear debris tapped within background fibrin clots (fig. 12a, b).

TP features:

(1) Malignant and keratinized cells

(2) Tumor diathesis is minimal to nonexistent and best located within fibrin clots

\section{Discussion}

In our institution, salivary gland aspirates are performed either by radiologists under ultrasound guidance for nonpalpable lesions or by surgeons for palpable lesions. When rapid assessment is requested, we perform air-dried Diff-Quik smears for immediate evaluation and alcohol-fixed smears for Pap stain. After every pass, the needle is rinsed in CytoLyt ${ }^{\circledR}$ and, based on the findings, a decision is made by the pathologist whether to prepare a TP slide, a cell block or both. A TP is frequently requested to insure a well-preserved Pap-stained slide, especially in bloody samples or when we expect a low-yield rinse that is insufficient for a cell block. However, when the surgeons perform the aspirates without rapid assessment, we request that they directly rinse the needle in CytoLyt after preparing direct smears and that at least one additional pass is dedicated to the CytoLyt rinse. In the laboratory, the sample is split and both a TP and a cell block (when possible) are prepared.

Because of the many recognized advantages of the TP methodology, it has gained popularity as an alternative to conventional processing techniques for exfoliative nongynecological samples, fluid and FNA. The idea of rinsing a sample in a stable transporting solution and sending it to a laboratory without the need for refrigeration is attractive to many laboratories, especially larger commercial laboratories with centralized specimen processing. Specimen processing is easy and personnel with minimal qualifications can perform it efficiently. Background-obscuring material such as blood or mucus is greatly reduced and consequently the TP is an easy method for screening and locating abnormal cells. Despite the controversy of utilizing this methodology in FNA due to the known artifacts and alteration of architecture it introduces [1], many laboratories still elect to process their FNA specimens by TP. This decision is greatly influenced by the need to minimize the number of slides prepared in highvolume laboratories to remain in compliance with the 
mandated 100 maximum slides per day that can be screened per cytotechnologist. Many laboratories, however, remain reluctant to implement $\mathrm{TP}$ for salivary glands, which are already challenging by CS due to its highly variable architecture, the significance of extracellular matrix and its interface with the epithelial component in establishing an accurate diagnosis.

In 1997, Michael and Hunter [1] published their experience with 120 FNAs, including 13 aspirates from the salivary gland that were obtained at the surgical bench and prepared by both TP and CS. They compared the cytological features between the two methodologies and highlighted many artifacts noticed on TP. Several differences were noted to affect the background cells and particles, extracellular material, architectural integrity, and changes at the cellular level. Many of the small cells, including red blood cells, lymphocytes and inflammatory cells, were filtered and minimally represented on the preparation. Blood cells remaining after the lysis of blood were represented on the TP and sometimes confused with real inflammation. Large fragments and clusters of cells were broken into smaller groups and single background cells were consequently relatively increased. Extracellular matrix was altered both in quality and quantity. At the cellular level, due to the fixation process, the cells appeared relatively smaller and tended to be more spherical, making the cell morphology harder to evaluate. Despite the excellent fixation, chromatin detail was not always crisp. The cytoplasm tended to be relatively denser in quality, although vacuolation and granularity remained obvious.

Al-Khafaji and Afify [5] reviewed a total of 134 salivary gland FNAs, including 74 with surgical follow-up; 50 were processed by TP alone and 24 by CS. They reported that the nondiagnostic rate for TP was double that for CS (16 vs. $8 \%$ ); 3 cases were falsely classified as negative on TP ( 2 acinic cell carcinomas and 1 low-grade mucoepidermoid carcinoma) and 1 case was falsely positive (pleomorphic adenoma diagnosed initially as carcinoma due to the presence of atypical cells without the myxoid stro$\mathrm{ma})$. Overall, the sensitivity and specificity for the TPprocessed cases were 96 and $88 \%$, respectively, while those for CS were $100 \%$. They also reported distortion of the architectural relation between the cellular component and the stromal elements as well as many of the artifacts previously reported in relation to TP. Mygdakos et al. [2] reported a similar experience in their review of 96 aspirates prepared by TP, including 6 from the salivary gland.

Parfitt et al. [3] reviewed 98 satisfactory cases with surgical follow-up. They found that an accurate diagnosis was rendered more frequently based on CS than TP (57 vs. $42 \%$, respectively). However, they noted that the diagnostic accuracy for cellular TP and CS was similar.

A recent study by Hipp et al. [4] reported their review of 369 FNA of salivary gland aspirates with neoplastic follow-up. Of these, 178 were processed by TP only and 191 were processed by CS only or in combination with TP. The nondiagnostic rate was $40 \%$ for TP and $18 \%$ for CS. More intermediate diagnoses were reported on TP (40\% compared to $26 \%$ for CS). However, the absolute cytohistological concordance rate for cases diagnosed definitively as positive (i.e. type of neoplasm specified) was similar ( 80 vs. $86 \%$, respectively).

In this review, we present our experience with salivary gland FNAs prepared by TP. Overall, it is important to recognize the nature of artifacts introduced by the TP both in general and those specific to FNA (table 2). Various differences exist between CS and TP, some due to the elimination of manual spreading and others related to the TP methodology, as detailed in table 1. However, based on the above studies, it is clear that these artifacts do not negatively influence the diagnostic accuracy when the aspirate is adequately cellular. It has been our experience that the more cellular the sample, the easier and more accurate is the diagnosis. While this would apply to any methodology, it is particularly important for TP as it increases the chances of recognizing the needed diagnostic features, particularly those related to architecture and cell-to-stroma interface.

While the nondiagnostic rate was high in all the reported studies, this can be attributed to the absence of onsite rapid assessment rather than TP itself. Based on the methodology itself, the machine applies vacuum to the vial until either a certain density on the filter/slide is achieved or until the specimen is depleted [6]. Consequently, a low-cellular aspirate will probably be equally or even better represented on TP than CS but may remain inadequate for diagnosis.

In conclusion, $\mathrm{TP}$ can be implemented for salivary gland aspirates without compromise in diagnostic accuracy provided that the samples are of adequate cellularity. Complementary cell blocks can be of great value since the architectural features are relatively maintained and can be used for immunostains when needed. Since evaluating TP requires experience with the process, it is prudent to train the pathologists using a set of cases prepared by both $\mathrm{TP}$ and CS before implementing the practice for patient care. 
References

Michael CW, Hunter B: Interpretation of fine-needle aspirates processed by the ThinPrep technique: cytologic artifacts and diagnostic pitfalls. Diagn Cytopathol 2000;23:613.

2 Mygdakos N, Nikolaidou S, Tzilivaki A, Tamiolakis D: Liquid-based preparation (LBP) cytology versus conventional cytology (CS) in FNA samples from breast, thyroid, salivary glands and soft tissues. Our experience in Crete (Greece). Rom J Morphol Embryol 2009;50:245-250.

3 Parfitt JR, McLachlin CM, Weir MM: Comparison of ThinPrep and conventional smears in salivary gland fine-needle aspiration biopsies. Cancer Cytopathol 2007;111:123-129.

$\checkmark 4$ Hipp J, Lee B, Specter ME, Jing X: Diagnostic yield of ThinPrep preparation in the assessment of fine-needle aspiration biopsy of salivary gland neoplasms. Diagn Cytopathol, in press.

5 Al-Khafaji BM, Afify AM: Salivary gland fine-needle aspiration using the ThinPrep ${ }^{\circledR}$ technique: diagnostic accuracy, cytologic artifacts and pitfalls. Acta Cytol 2001;45:567574.

6 Operator's Manual: ThinPrep Processor. Marlborough, Cytyc, 1992. 